\section{Dementia care without drugs}

O'Callaghan et al (2010) rightly highlight the fact that non-pharmacological management is a realistic intervention to reduce the risk of aggression and violence in elderly people with dementia. The NICE clinical guideline on dementia mentions individually tailored care plans and consideration of approaches such as aromatherapy, multisensory stimulation, therapeutic use of music and/or dancing, animal-assisted therapy and massage to reduce agitation and aggression (National Institute for Health and Clinical Excellence 2006).

I remember during my MRCPsych CASC examination that I had to explain these nonpharmacological interventions to a patient's relative, who seemed very pleased and relieved that there were alternatives to medication. However, numerous hospitals and care homes already do not provide such treatments. And in the current economic climate, with NHS and social care facing many cuts in the near future, I wonder whether elderly patients will continue to have access to these very useful therapies in services that offer them at present. Losing access to these therapies could have a detrimental effect on our patients' physical and mental health. As mental health professionals, I think we have a role in highlighting the importance of these approaches: not only are they of great benefit to our patients, but also they reduce the risk of violence, making things safer for patients, staff and carers.

National Institute for Health and Clinical Excellence (2006) Dementia. A NICE-SCIE Guideline on Supporting People with Dementia and their Carers in Health and Social Care (Clinical Guideline CG42). The British Psychological Society \& Gaskell.

O'Callaghan CE, Richman AV, Majumdar B (2010) Violence in older people with mental illness. Advances in Psychiatric Treatment 16: 339-48.

Gagan Preeti, CT3, South West London and St George's Mental Health Trust, London, UK. Email: Gagan.Preeti@swlstg-tr.nhs.uk doi: 10.1192/apt.17.3.236

\section{Can we do it?}

Psychiatry has always been a poor sister in comparison with general medicine. In the early 1900s, psychiatry and mental illness were already attracting negative coverage in the media, and things have not changed much since, despite the digital revolution.

We cannot understand the apathy within psychiatry itself in the face of this: instead of taking the media head on about its negative bias, psychiatrists have failed to stop it. Every day, news stories are flashed out about people with mental disorder, but rather than challenging the negative thinking about our clients, we become entangled in a blame game against psychiatry itself.

The stigma surrounding mental illness is here to stay unless we as psychiatrists start to work on attitudes in our own workplace and on the apathy that seems to have submerged the field.

The media remains more interested in sensational stories than in working with psychiatry and its professionals to change misconceptions.

They say it is never too late to start a revolution. But who will do it?

Bithell C (2011) Why psychiatry should engage with the media. Advances in Psychiatric Treatment 17: 82-4.

A. K. J. Shishodia, Locum ST4, Greater Manchester West Foundation NHS Trust; P. Tandon, Locum ST4, Manchester Mental Health NHS Trust, Manchester, UK. Email: ashokshishodia@yahoo. co.uk

doi: 10.1192/apt.17.3.236a

\section{Significance of MCQs for trainees}

The MCQs at the end of articles in Advances are very valuable for trainees, particularly as papers 1, 2 and 3 of the MRCPsych consist of MCQs and EMIs. Practising a lot of questions is an important aspect of revising for the Membership examinations, and having MCQs with each article is certainly helpful.

Bouch J (2010) MCQs and learning. Advances in Psychiatric Treatment 16: 237.

Gagan Preeti, CT3, South West London and St George's Mental Health Trust, London, UK. Email: Gagan.Preeti@swlstg-tr.nhs.uk doi: 10.1192/apt.17.3.236b

\section{A conundrum about confidence intervals}

The article by Hodgson et al (2011) unfortunately glosses over a problem with confidence intervals and numbers needed to treat. The problem is that, when the result is not significant, the confidence interval will include infinity (Altman 1998) (since the absolute risk difference at zero implies a number needed to treat of 1 divided by zero, which is infinity). This is a mathematical conundrum that detracts from any medical interpretation and can confuse forest plots.

Some statisticians are suspicious of the number needed to treat, although it is preferred by many clinicians. The consensus seems to be that the number needed to treat is less easy to understand 
without an emphasis on the underlying absolute risk difference. The number needed to treat is not as efficient an indicator of effect size as are the odds ratio or relative risk in meta-analyses.

Clinicians have to navigate with much difficulty through the statistical fog of evidence-based medicine. There is a lack of articles such as that by Hodgson et al and one can often feel shipwrecked on the mathematical complexities of concepts such as the number needed to treat.
Altman DG (1998) Confidence intervals for the number needed to treat. BMJ 317: 1309-12.

Hodgson R, Cookson J, Taylor M (2011) Numbers-needed-to-treat analysis: an explanation using antipsychotic trials in schizophrenia. Advances in Psychiatric Treatment 17: 63-71.

Zekria Ibrahimi, psychiatric patient, Coombs Library, West London Mental Health Trust, Southall UB1 3EU, UK. Email: ibrahimizekria@googlemail.com

doi: 10.1192/apt.17.3.236c 